\title{
Two separate dose-dependent effects of paroxetine: mydriasis and inhibition of tramadol's $O$-demethylation via CYP2D6
}

\author{
Anette Green Nielsen • Rasmus Steen Pedersen • \\ Lene Noehr-Jensen • Per Damkier • Kim Brosen
}

Received: 12 November 2009 / Accepted: 17 February 2010 /Published online: 31 March 2010

(C) Springer-Verlag 2010

\begin{abstract}
Purpose To investigate paroxetine's putative dose-dependent impact on pupil reaction and inhibition of the $O$-demethylation of tramadol.

Methods Twelve healthy CYP2D6 extensive metabolizers participated in this double-blinded randomized five-way placebo controlled cross-over study; they received placebo, $10,20,30$, and $50 \mathrm{mg}$ paroxetine as single oral doses at bedtime. Next morning the pupil was measured followed by oral intake of $50 \mathrm{mg}$ of tramadol, and urine was collected for $8 \mathrm{~h}$. Three hours after ingestion of tramadol a second measurement of the pupil was performed. Enantioselective urine concentrations of (+/-)-tramadol and (+/-)$O$-desmethyltramadol (M1) were determined.

Results With placebo, the median maximum pupil diameter was $6.43 \mathrm{~mm}$ (range $5.45-7.75 \mathrm{~mm}$ ) before tramadol and $6.22 \mathrm{~mm}(4.35-7.65 \mathrm{~mm})$ after $50 \mathrm{mg}$ of tramadol $(P=0.4935)$. Paroxetine resulted in a statistically significant, dosedependent dilatation of the pupil with a geometric mean difference of 1.17 (95\% CI 1.10-1.24) after ingestion of $50 \mathrm{mg}$ paroxetine $(P<0.001)$. Likewise, a reduction in the relative constriction amplitude with a geometric mean difference of $0.81(95 \%$ CI $0.71-0.92)(P<0.001)$ was seen. A dose-dependent inhibition of the metabolism of tramadol by an increase in the two urinary metabolic ratios $(+)$-tramadol / (+)-M1 [geometric mean difference 9.09,
\end{abstract}

A. G. Nielsen • R. S. Pedersen $(\square) \cdot$ L. Noehr-Jensen • K. Brosen Institute of Public Health, Clinical Pharmacology,

University of Southern Denmark,

Odense, Denmark

e-mail: rpedersen@health.sdu.dk

\section{P. Damkier}

Department of Biochemistry, Pharmacology \& Genetics,

Odense University Hospital,

Odense, Denmark
95\% CI 5.60-14.73 $(P<0.001)]$ and $(-)-\mathrm{M} 1 /(+)-\mathrm{M} 1$ [geometric mean difference $2.84,95 \%$ CI 2.15-3.77 $(P<$ 0.001)] was also observed.

Conclusions Paroxetine is a dose-dependent dilator of the pupil and as expected a dose-dependent inhibitor of $(+)$ tramadol's $O$-demethylation.

Keywords Paroxetine - Tramadol - Interaction · CYP2D6 . Pupillometry $\cdot$ Metabolic ratios

\section{Introduction}

Chronic pain and depression show considerable co-morbidity [1], and each of the two conditions often requires treatment with drugs. Thus drug-drug interactions between analgesics and antidepressants are an important medical issue. Tramadol is a synthetic opioid used for mild to moderate pain [2]. Tramadol is a racemate consisting of (+)-tramadol hydrochloride and (-)-tramadol hydrochloride. These compounds are metabolized to $(+)$ and $(-)-O$-desmethyltramadol $[(+)-\mathrm{M} 1$ and (-)-M1] and both reactions, but in particular the former, are catalyzed by CYP2D6 [3-8]. (+)-M1 is the active $\mu$-opioid receptor agonist, while (+)-tramadol is an inhibitor of the 5-HT re-uptake and (-)-tramadol inhibits norepinephrine re-uptake [9-12].

CYP2D6 is involved in the metabolism of about $15 \%$ of the medical substances that are metabolized by the CYP enzymes [13]. CYP2D6 polymorphism divides the population into two phenotypes: individuals without catalytic CYP2D6 activity, i.e., poor metabolizers (PMs), and individuals with present but variable catalytic CYP2D6 activity, i.e., extensive metabolizers (EMs). About $7-8 \%$ of the Caucasian population has the phenotype PM $[14,15]$. The impaired $O$-demethylation of (+)-tramadol in the PM 
results in less (+)-M1 being formed and an impaired analgesic effect in this phenotype compared with the EM [5]. Paroxetine is a selective serotonin reuptake inhibitor (SSRI) that is metabolized by CYP2D6 $[16,17]$ and also an inhibitor of the enzyme [16-21]. Paroxetine seems to be a mechanism-based inhibitor of CYP2D6 [22]. Paroxetine shows nonlinear saturation of CYP2D6 at steady-state plasma concentrations. At steady state, daily doses of 20 $30 \mathrm{mg}$ paroxetine result in a reduction of CYP2D6 activity by about $93 \%$ [23].

Tramadol, like other opioids, causes a dose-dependent miosis through the active metabolite $(+)-\mathrm{M} 1$, which is caused by an excitatory effect on the parasympathetic nervous system [24]. Through the inhibition by paroxetine of the formation of (+)-M1, one would expect that the pupil contraction caused by tramadol would diminish when administered together with paroxetine and that both effects would be dose-dependent.

\section{Methods}

\section{Subjects}

Twelve healthy volunteers, aged $22-32$ years, participated in this study, including nine males and three females. All subjects were CYP2D6 EMs as assessed by previous phenotype studies $[25,26]$. Female subjects conducted a negative pregnancy test prior to the beginning of the study and used safe contraception thoughout the trial. Routine laboratory tests (blood chemistry and hematology) were within normal values. Physical examination and medical history did not reveal signs of disease, drug abuse or alcohol dependence. Concomitant medication was not allowed.

Study design

The trial was registered in the European Clinical Trials Database (EudraCT no. 2008-001516-20) and in the US National Institutes of Health registry at http://www. clinicaltrials.gov (NCT 00785603). The protocol was approved by the Danish Medicines Agency (J. no. 26123730), the Local Ethics Committee for the Region of Southern Denmark (J.no. S-20080043) and the Danish Data Protection Agency (J.no. 2008-41-2277). The trial was carried out in accordance with Good Clinical Practice (GCP) guidelines and monitored by the GCP Unit, Odense University Hospital.

Sample size calculations were based on a primary outcome represented by interindividual differences in pupil diameter of subjects receiving 10 and $50 \mathrm{mg}$ paroxetine respectively. It was estimated that a difference of one standard deviation could be detected given a two-sided level of significance $(\alpha)$ of 0.05 and a power of $80 \%$ using ten subjects. To allow for a possible drop out rate of $15 \%$, 12 subjects were recruited.

The study was conducted as a double-blinded, randomized, five-way, placebo controlled, cross-over study. Subjects went through five phases receiving a single dose of 10, 20, 30, $50 \mathrm{mg}$ paroxetine (Paroxetin, Copyfarm, Denmark) or placebo, respectively, before bedtime. The following day a measurement of the pupil size was performed followed by administration of $50 \mathrm{mg}$ tramadol (Nobligan ${ }^{\circledR}$, Grünenthal, Germany). Three hours later, the second measurement of the pupil was performed. The subjects collected urine for $8 \mathrm{~h}$ after ingestion of tramadol. The five phases were separated by a wash out period of minimum 2 weeks. Packing, blinding, and randomization of the drugs was conducted by Odense University Hospital Pharmacy.

Pupil measurement

The measurement of the pupil was performed with a handheld pupillometer PLR-100 ${ }^{\mathrm{TM}}$, NeurOptics Inc. The light intensity was held constant at $25 \mathrm{~cd} / \mathrm{m}^{2}$ for all the pupil measurements, and the same eye of each subject was measured. The subjects adjusted to the light for 2 min and then focused on a spot on the wall $3 \mathrm{~m}$ away. After a focus period of $3 \mathrm{~s}$, a measure period of $3.2 \mathrm{~s}$ followed during which a flash of light was emitted and the pupillary reaction was measured. The results of a measurement consisted of the maximum and the minimum pupil diameter. From these the more robust measure of relative constriction amplitude was calculated as (max. diameter $-\min$. diameter) / max. diameter.

\section{Sample analysis}

In all phases, total urine volumes were registered and aliquots were stored at $-20^{\circ} \mathrm{C}$ until analysis. Concentrations of $(+)-$ tramadol and (-)-tramadol and the metabolites (+)-M1 and (-)M1 in the urine were determined by enantioselective high performance liquid chromatography (HPLC) as described in detail in our previous method article [27]. We have previously shown that tramadol is a validated probe for CYP2D6 phenotyping [28]. Two urinary metabolic ratios can be determined from urine collected over $8 \mathrm{~h}$ after ingestion of $50 \mathrm{mg}$ tramadol. The first metabolic ratio $\left(\mathrm{MR}_{1}\right)$ is calculated as the amount of excreted $(+)$-tramadol divided by the amount of excreted metabolite (+)-M1. The second metabolic ratio $\left(\mathrm{MR}_{2}\right)$ is calculated as the amount of excreted $(-)$-M1 divided by the amount of excreted (+)-M1. MR 2 is the most valid measurement for determining the phenotype of CYP2D6 by using tramadol. EMs have an $\mathrm{MR}_{2}<2.0$, and $\mathrm{PMs}$ have an $\mathrm{MR}_{2} \geq 2.0$ [28]. 


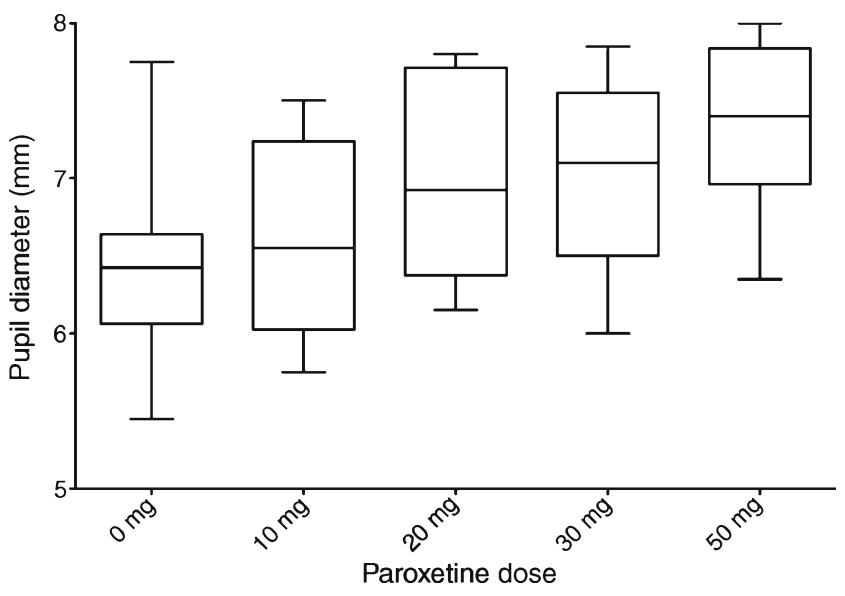

Fig. 1 Median maximum pupil diameter with $25-75 \%$ percentile and range for each dose of paroxetine measured at baseline before administration of tramadol

\section{Statistical analysis}

Data are presented as medians with $25-75 \%$ percentile and range. Statistic inference was analyzed by means of paired $t$-test and repeated measures one-way ANOVA. ANOVA was followed by a Tukey's multiple comparison test. The data were transformed by the natural logarithm prior to statistical analysis in order to justify Gaussian distribution.

\section{Results}

Eleven subjects completed all five phases and one subject dropped out after four phases thereby missing the phase requiring ingestion of $30 \mathrm{mg}$ paroxetine. The most frequently reported adverse events were fatigue $(n=3)$, sleep disturbances $(n=5)$, nausea $(n=6)$, and anxiety $(n=4)$.

The results from the subject that did not complete the last phase were not included in the repeated measures ANOVA and the Tukey's multiple comparison test but only presented in the figures illustrating the descriptive data.

The effect of tramadol on the pupil

The effect of tramadol on the pupil is seen by analyzing the difference between the results of the first and the second pupil measurement when $0 \mathrm{mg}$ paroxetine (=placebo) was administered. The first measured median maximum pupil diameter was $6.43 \mathrm{~mm}$ (range $5.45-7.75 \mathrm{~mm}$ ) and the second measured median maximum pupil diameter was $6.22 \mathrm{~mm}$ (range 4.35$7.65 \mathrm{~mm}) 3 \mathrm{~h}$ after ingesting $50 \mathrm{mg}$ of tramadol. The paired $t$-test carried out on the logarithmic values showed no statistically significant difference between the two pupil measurements $(P=0.4935)$. The first measured median relative constriction amplitude was $0.395 \mathrm{~mm}$ (range $0.252-$ $0.464 \mathrm{~mm}$ ) and the second measured median relative

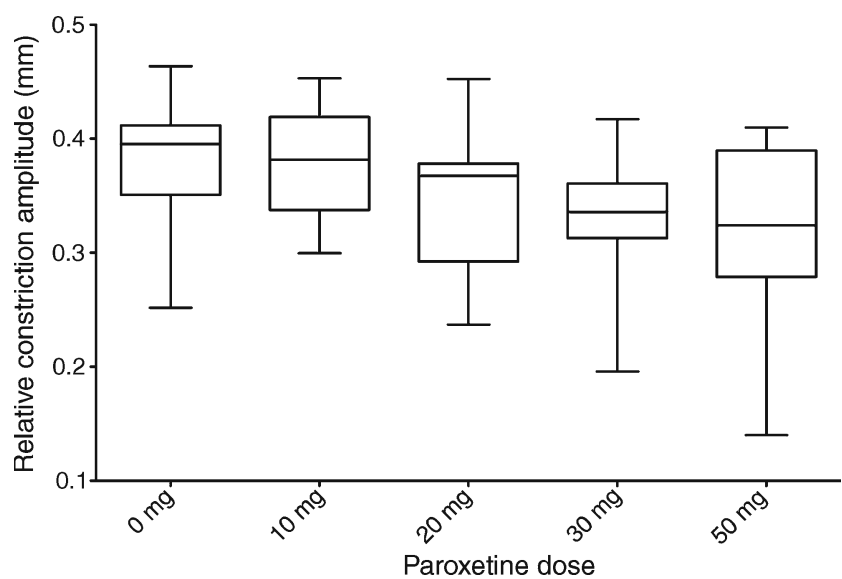

Fig. 2 Median relative restriction amplitude with $25-75 \%$ percentile and range for each dose of paroxetine measured at baseline before administration of tramadol

constriction amplitude was $0.375 \mathrm{~mm}$ (range $0.318-$ $0.416 \mathrm{~mm})$. No statistical significance was found $(P=0.0954)$

There was no statistically significant difference between the two measurements at any of the other phases with administration of concomitant paroxetine (data not shown). There was no correlation between the amounts of excreted (+)-M1 and pupil measurements either (data not shown). Overall, this shows that $50 \mathrm{mg}$ tramadol has no effect on the pupil, and it was therefore not possible to observe a reduction in the miotic effect of tramadol with contemporary administration of different doses of paroxetine.

The effect of paroxetine on the pupil

The result that shows the exclusive effect of paroxetine on the pupil is the first measurement of the pupil in each phase as the subjects had not yet ingested tramadol at this point. The

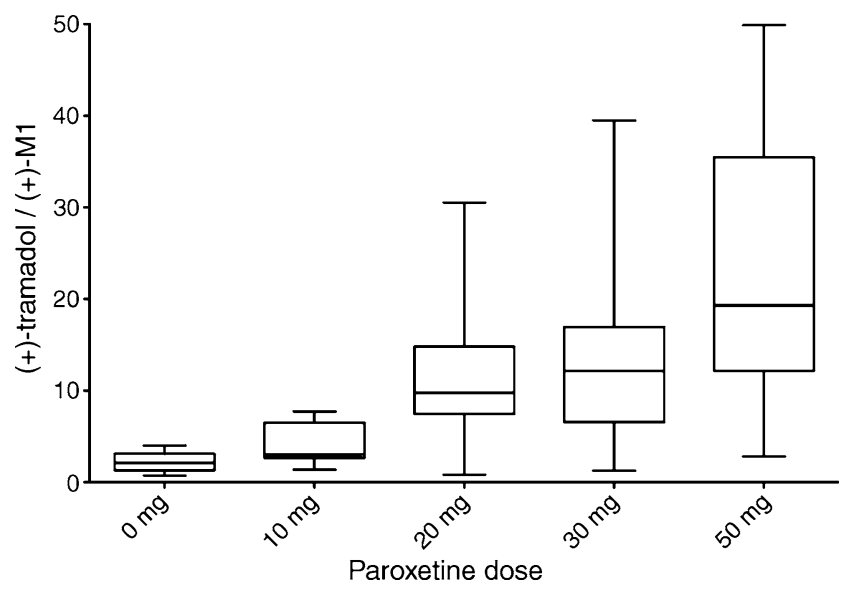

Fig. 3 Median $\mathrm{MR}_{1}=$ amount of excreted (+)-tramadol / amount of excreted (+)-M1 with $25-75 \%$ percentile and range for each dose of paroxetine 


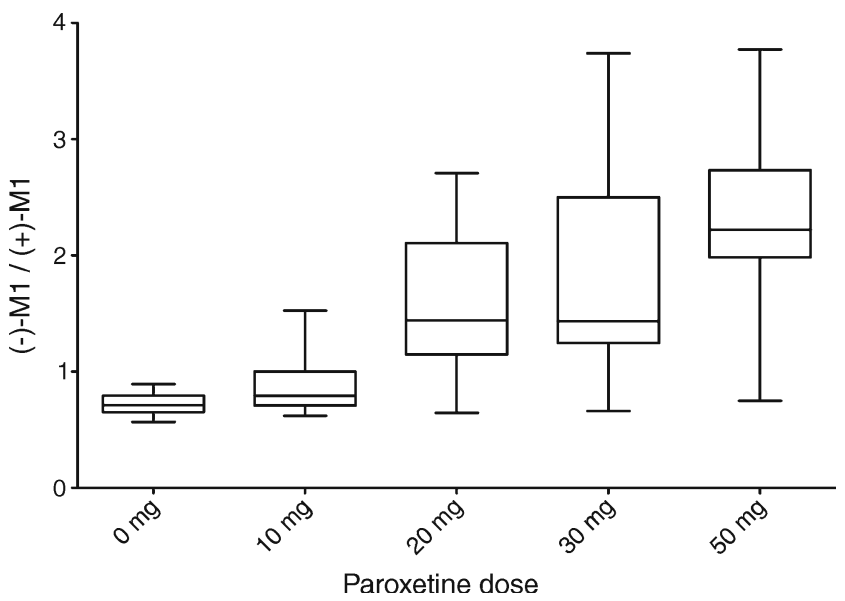

Fig. 4 Median $\mathrm{MR}_{2}=$ amount of excreted (-)-M1 / amount of excreted $(+)-\mathrm{M} 1$ with $25-75 \%$ percentile and range for each dose of paroxetine

median maximum pupil diameter with $25-75 \%$ percentile and range is shown for each phase in Fig. 1. The clear increase in the maximum pupil diameter with increasing doses of paroxetine was confirmed by repeated measures ANOVA $(P<0.0001)$ and a subsequent Tukey's test as presented in Table 1. The ingestion of $50 \mathrm{mg}$ paroxetine resulted in a change in geometric mean difference of 1.17 (95\% CI 1.101.24) compared to the placebo phase.

Likewise, the relative constriction amplitude decreased significantly with increasing dose of paroxetine $(P<0.0001)$ as shown in Fig. 2. The geometric mean difference was 0.81 (95\% CI 0.71-0.92) in the phase with $50 \mathrm{mg}$ paroxetine compared to the placebo phase.

The effect of paroxetine on metabolic ratios

The median metabolic ratio $\mathrm{MR}_{1}$ with $25-75 \%$ percentile and range is shown for each phase in Fig. 3. A clear stepwise increase in median is seen with increasing dose of paroxetine. The repeated measures ANOVA was statistically significant $(P<0.0001)$, and the subsequent Tukey's test including geometric mean difference with $95 \%$ CI carried out on the values of $M_{1}$ is shown in Table 1 . The $M_{1}$ geometric mean difference between the placebo phase and the highest dose of $50 \mathrm{mg}$ paroxetine was 9.09 with $95 \% \mathrm{CI}$ of 5.60-14.73. In fact, all dosing steps resulted in statistically significant changes in $\mathrm{MR}_{1}(P<0.05)$ apart from the step from 20 to $30 \mathrm{mg}$ paroxetine.

As shown in Fig. 4 and Table 1 , the $\mathrm{MR}_{2}$ also increased with increasing dose of paroxetine. The repeated measures ANOVA shows likewise a significant increase in $\mathrm{MR}_{2}(P<0.0001)$. In this case a geometric mean difference of 2.84 with $95 \%$ CI of $2.15-3.77$ resulted in 10 out of 12 subjects changing phenotype from EM to PM by ingestion of $50 \mathrm{mg}$ paroxetine according to the validated CYP2D6 phenotype marker $\mathrm{MR}_{2}$.

To summarize, the dosing steps from placebo and from $10 \mathrm{mg}$ paroxetine to 30 and to $50 \mathrm{mg}$ paroxetine resulted in statistically significant alterations in both the dynamic and kinetic parameters: maximum pupil size, relative restriction amplitude, $\mathrm{MR}_{1}$, and $\mathrm{MR}_{2}$.

\section{Discussion}

The aim of the study was to investigate the effect of an inhibition of CYP2D6 caused by paroxetine on the pharmacodynamics and pharmacokinetics of tramadol.

In this study we demonstrate that the maximum pupil diameter is increased and the relative constriction amplitude is decreased with increasing dose of paroxetine. Both are caused by an anticholinergic effect [29]. To our knowledge we are the first to report this clear dose-dependent mydriasis

Table 1 Geometric mean ratios (95\% CI) between each dose of paroxetine

\begin{tabular}{|c|c|c|c|c|}
\hline Tukey's multiple comparison test & Maximum pupil diameter & Relative constriction amplitude & $\mathrm{MR}_{1}^{\mathrm{a}}$ & $\mathrm{MR}_{2}{ }^{\mathrm{b}}$ \\
\hline $0 \mathrm{mg}$ vs $10 \mathrm{mg}$ & $1.05(0.99-1.11)$ & $0.98(0.86-1.12)$ & $1.81(1.12-2.93)^{* *}$ & $1.20(0.91-1.59)$ \\
\hline $0 \mathrm{mg}$ vs $20 \mathrm{mg}$ & $1.11(1.05-1.18)^{* * *}$ & $0.89(0.78-1.02)$ & $4.29(2.64-6.94)^{* * *}$ & $2.00(1.51-2.66)^{* * *}$ \\
\hline $0 \mathrm{mg}$ vs $30 \mathrm{mg}$ & $1.12(1.06-1.19)^{* * *}$ & $0.82(0.72-0.94)^{* *}$ & $5.40(3.33-8.76)^{* *}$ & $2.27(1.71-3.01)^{* * *}$ \\
\hline $0 \mathrm{mg}$ vs $50 \mathrm{mg}$ & $1.17(1.10-1.24)^{* * *}$ & $0.81(0.71-0.92)^{* * *}$ & $9.09(5.60-14.73)^{* * *}$ & $2.84(2.15-3.77)^{* * *}$ \\
\hline $10 \mathrm{mg}$ vs $20 \mathrm{mg}$ & $1.06(1.00-1.13)^{*}$ & $0.91(0.80-1.04)$ & $2.37(1.46-3.83)^{* * *}$ & $1.67(1.26-2.21)^{* * *}$ \\
\hline $10 \mathrm{mg}$ vs $30 \mathrm{mg}$ & $1.07(1.01-1.14)^{*}$ & $0.84(0.74-0.96)^{* *}$ & $2.98(1.84-4.84)^{* * *}$ & $1.89(1.42-2.51)^{* * *}$ \\
\hline $10 \mathrm{mg}$ vs $50 \mathrm{mg}$ & $1.12(1.05-1.18)^{* * *}$ & $0.83(0.73-0.95)^{* * *}$ & $5.01(3.10-8.13)^{* * *}$ & $2.37(1.78-3.14)^{* * *}$ \\
\hline $20 \mathrm{mg}$ vs $30 \mathrm{mg}$ & $1.01(0.95-1.07)$ & $0.92(0.81-1.05)$ & $1.26(0.78-2.04)$ & $1.13(0.86-1.50)$ \\
\hline $20 \mathrm{mg}$ vs $50 \mathrm{mg}$ & $1.05(0.99-1.11)$ & $0.91(0.79-1.04)$ & $2.12(1.31-3.44)^{* * *}$ & $1.42(1.07-1.88)^{* * *}$ \\
\hline $30 \mathrm{mg}$ vs $50 \mathrm{mg}$ & $1.04(0.98-1.10)$ & $0.99(0.86-1.13)$ & $1.68(1.03-2.73)^{*}$ & $1.25(0.94-1.66)$ \\
\hline
\end{tabular}

${ }^{\mathrm{a}} \mathrm{MR}_{1}=$ amount of excreted (+)-tramadol / amount of excreted (+)-M1

${ }^{\mathrm{b}} \mathrm{MR}_{2}=$ amount of excreted (-)-M1 / amount of excreted (+)-M1

${ }^{*} P<0.05,{ }^{*} * P<0.01, * * * P<0.001$ 
induced by a single dose of paroxetine. Dilatation of the pupil is stated to be an uncommon adverse event for paroxetine [30] and has been reported in the clinic [31]. It has also previously been reported that a single dose of $20 \mathrm{mg}$ paroxetine did not cause any significant effect on the pupil diameter [32]. In the study by Bitsios, the pupil diameter was measured $3 \mathrm{~h}$ after ingestion of paroxetine [32]. As paroxetine achieves a maximum plasma concentration $6 \mathrm{~h}$ after oral administration [30], a pupil measurement after $3 \mathrm{~h}$ is unlikely to reveal the full effect of paroxetine on the pupil diameter. In the current study the first pupil diameter measurement was performed approximately $9 \mathrm{~h}$ after administration of paroxetine, where an effect is biologically more plausible. Paroxetine plasma concentrations would perhaps have elucidated this issue. Furthermore, the correlation between paroxetine plasma concentrations and the endpoints could have been investigated. Unfortunately this was not included in the design of the study and therefore not feasible. On the other hand, the volunteers were a homogeneous group of healthy CYP2D6 EMs who probably display very little interindividual paroxetine PK. This assumption was fortunately supported by the clear correlation between paroxetine dose and both the dynamic and the kinetic end points.

We have recently shown that both a single and multiple doses of $10 \mathrm{mg}$ of another SSRI, escitalopram, also caused a decrease in the relative constriction amplitude whereas no effect on the maximum pupil diameter was seen [33]. However a subsequent study showed that daily intake of $20 \mathrm{mg}$ of escitalopram increased the maximum pupil diameter and decreased the relative constriction amplitude [26]. This mydriatic effect is therefore probably a class effect of the SSRIs having affinity for muscarinic cholinergic receptors [31].

This study did not show any effect of $50 \mathrm{mg}$ single oral dose tramadol on the pupil diameter and subsequently a reduction in the pupil contraction caused by tramadol during concomitant administration of paroxetine could not be demonstrated. The most likely explanation is that a dose of $50 \mathrm{mg}$ tramadol is too low to cause contraction of the pupil. It has previously been shown that the lowest dose of tramadol to cause miosis is $100 \mathrm{mg}$ [24]. Due to the demonstrated opposite effect of paroxetine on the pupil, it is unclear whether a dose of $100 \mathrm{mg}$ tramadol in the present study would have resulted in a measurable pharmacodynamic change related to the pharmacokinetic interaction.

This study also clearly demonstrates that paroxetine is, as expected [34], a dose-dependent inhibitor of tramadol's $O$-demethylation via CYP2D6. This result also serves as yet another validation criterion of this CYP2D6 phenotype test. Any cytochrome P450 phenotyping metric should display sensitivity toward drug-induced inhibition [35], which is exactly what this study shows.
We have previously shown that daily intake of $20 \mathrm{mg}$ paroxetine inhibited the formation of $(+)-\mathrm{M} 1$ and reduced the analgesic effect of tramadol [6]. By extrapolation we hypothesize that the reduction in tramadol's hypoalgesic effect caused by paroxetine is also dose-dependent, and the combination of the two drugs should be avoided.

Acknowledgments We thank the Danish Research Council for Health and Disease; grant number 271-05-0266. We also thank Birgitte Damby Sorensen for analytical expertise.

Competing interests The head of the research group, K.B., is a member of the Advisory board of the Lundbeck Institute and has previously received funds for members of staff from $\mathrm{H}$. Lundbeck $\mathrm{A} / \mathrm{S}$.

\section{References}

1. Bair MJ, Robinson RL, Katon W, Kroenke K (2003) Depression and pain comorbidity. Arch Intern Med 163:243-245

2. Trescot AM, Datta S, Lee M, Hansen H (2008) Opioid pharmacology. Pain Physician 11:133-153, Opioid Special Issue

3. Paar WD, Frankus P, Dengler HJ (1992) The metabolism of tramadol by human liver microsomes. Clin Investig 70:708-710

4. Paar WD, Poche S, Gerloff J, Dengler HJ (1997) Polymorphic CYP2D6 mediates O-demethylation of the opioid analgesic tramadol. Eur J Clin Pharmacol 53:235-239

5. Poulsen L, Arendt-Nielsen L, Brosen K, Sindrup SH (1996) The hypoalgesic effect of tramadol in relation to CYP2D6. Clin Pharmacol Ther 60:636-644

6. Laugesen S, Enggaard TP, Pedersen RS, Sindrup SH, Brosen K (2005) Paroxetine, a cytochrome P450 2D6 inhibitor, diminishes the stereoselective $O$-demethylation and reduces the hypoalgesic effect of tramadol. Clin Pharmacol Ther 77:312-323

7. Pedersen RS, Damkier P, Brosen K (2006) Enantioselective pharmacokinetics of tramadol in CYP2D6 extensive and poor metabolizers. Eur J Clin Pharmacol 62:513-521

8. Halling J, Weihe P, Brosen K (2008) CYP2D6 polymorphism in relation to tramadol metabolism: a study of Faroese patients. Ther Drug Monit 30:271-275

9. Raffa RB, Friederichs E, Reimann W, Shank RP, Codd EE, Vaught JL (1992) Opioid and nonopioid components independently contribute to the mechanism of action of tramadol, an atypical opioid analgesic. J Pharmacol Exp Ther 260:275-285

10. Lai J, Ma SW, Porreca F, Raffa RB (1996) Tramadol, M1 metabolite and enantiomer affinities for cloned human opioid receptors expressed in HN9.10 neuroblastoma cells. Eur J Pharmacol 316:369-372

11. Bamingbade TA, Davidson C, Langford RM, Stamford JA (1997) Actions of tramadol, its enantiomers and principal metabolite, O-desmethyltramadol, on serotonin (5-HT) efflux and uptake in the rat dorsal raphe nucleus. Br J Anaesth 79:352-356

12. Halfpenny DM, Callado LF, Hopwood SE, Bamigbade TA, Langford RM, Stamford JA (1999) Effects of tramadol stereoisomers on norepinephrine efflux and uptake in the rat locus coeruleus measured by real time voltammetry. Br J Anaesth 83:909-915

13. Zanger UM, Turpeinen M, Klein K, Schwab M (2008) Functional pharmacogenetics/genomics of human cytochromes $\mathrm{P} 450$ involved in drug biotransformation. Anal Bioanal Chem 392:1093-1108

14. Ingelman-Sundberg M, Sim SC, Gomez A, Rodriguez-Antona C (2007) Influence of cytochrome P450 polymorfisms on drug therapies: pharmacogenetic, pharmacoepigenetic and clinical aspects. Pharmacol Ther 116:496-526 
15. Ingelman-Sundberg M (2005) Genetic polymorphisms of cytochrome P450 2D6 (CYP2D6): clinical consequences, evolutionary aspects and functional diversity. Pharmacogenom J 5:6-13

16. Sindrup SH, Brosen K, Gram LF (1992) Pharmacokinetics of the selective serotonin reuptake inhibitor paroxetine: nonlinearity and relation to the sparteine oxidation polymorphism. Clin Pharmacol Ther 51:288-295

17. Sindrup SH, Brosen K, Gram LF, Hallas J, Skjelbo E, Allen A, Allen GD, Cooper SM, Mellows G, Tasker TC et al (1992) The relationship between paroxetine and the sparteine oxidation polymorphism. Clin Pharmacol Ther 51:278-287

18. Brosen K, Gram LF, Kragh-Sørensen P (1991) Extremely slow metabolism of amitriptyline but normal metabolism of imipramine dnd Desipramine in an extensive metabolizer of sparteine, debrisoquine, and mephenytoin. Ther Drug Monit $13: 177-182$

19. Brosen K, Hansen JG, Nielsen KK, Sindrup SH, Gram LF (1993) Inhibition by paroxetine of desipramine metabolism in extensive but not in poor metabolizers of sparteine. Eur J Clin Pharmacol 44:349-355

20. Skjelbo E, Brosen K (1992) Inhibitors of imipramine metabolism by human liver microsomes. Br J Clin Pharmacol 34:256-261

21. Crewe HK, Lennard MS, Tucker GT, Woods FR, Haddock RE (1992) The effect of selective serotonin re-uptake inhibitors on cytochrome P4502D6 (CYP2D6) activity in human liver microsomes. Br J Clin Pharmacol 34:262-265

22. Bertelsen KM, Venkatakrishnan K, Moltke L, Obach RS, Greenblatt DJ (2003) Apparent mechanism-based inhibition of human CYP2D6 in vitro by paroxetine: comparison with fluoxetine and quinidine. Drug Metab Disp 31:289-293

23. Venkatakrishnan K, Obach RS (2005) In vitro-in vivo extrapolation of CYP2D6 inactivation by paroxetine: prediction of nonstationary pharmacokinetics and drug interaction magnitude. Drug Metab Disp 33:845-852

24. Fliegert F, Kurth B, Göhler (2005) The effects of tramadol on static and dynamic pupillometry in healthy subjects - the relationship between pharmacodynamics, pharmacokinetics and CYP2D6 metaboliser status. Eur J Clin Pharmacol 61:257-266
25. Zwisler ST, Enggaard TP, Noehr-Jensen L, Pedersen RS, Mikkelsen S, Nielsen F, Brosen K, Sindrup SH (2009) The hypoalgesic effect of oxycodone in human experimental pain models in relation to the CYP2D6 oxidation polymorphism. Basic Clin Pharmacol Toxicol 104:335-344

26. Noehr-Jensen L, Zwisler ST, Larsen F, Sindrup SH, Damkier P, Brosen K (2009) Escitalopram is a weak inhibitor of the CYP2D6-catalyzed O-demethylation of $(+)$-tramadol but does not reduce the hypoalgesic effect in experimental pain. Clin Pharmacol Ther 86:626-633

27. Pedersen RS, Brosen K, Nielsen F (2003) Enantioselective HPLC method for quantitative determination of tramadol and $\mathrm{O}$ desmethyltramadol in plasma and urine: application to clinical studies. Chromatographia 57:279-285

28. Pedersen RS, Damkier P, Brosen K (2005) Tramadol as a new probe for cytochrome P450 2D6 phenotyping: a population study. Clin Pharmacol Ther 77:458-467

29. Johnson AM (1989) An overview of the animal pharmacology of paroxetine. Acta Psychia Scan 80:14-20

30. Danish Medicines Agency (2009) Product resume for Seroxat ${ }^{\mathbb{R}}$. http://www.produktresume.dk. Accessed June 18, 2009

31. Larson M, Folstein S (2000) Selective serotonin reuptake inhibitorinduced mydriasis. J Am Acad Child Adolesc Psych 39:138-139

32. Bitsios P, Szabadi E, Brandshaw CM (1999) Comparison of the effects of venlafaxine, paroxetine and desipramine on the pupillary light reflex in man. Psychopharmacology 143:286-292

33. Noehr-Jensen L, Zwisler ST, Larsen F, Sindrup SH, Damkier P, Nielsen F, Brosen K (2009) Impact of CYP2C19 phenotypes on escitalopram metabolism and an evaluation of pupillometry as a serotonegic biomarker. Eur J Clin Pharmacol 65:887-894

34. Jeppesen U, Gram LF, Vistisen K, Loft H, Poulsen HE, Brosen K (1996) Dose-dependent inhibition of CYP1A2, CYP2C19 and CYP2D6 by citalopram, fluoxetine, fluvoxamine and paroxetine. Eur J Clin Pharmacol 51:73-78

35. Fuur U, Jetter A, Kirchheiner J (2007) Appropriate phenotyping procedures for drug metabolizing enzymes and transporters in humans and simultaneous use in the "cocktail approach". Clin Pharmacol Ther 81:270-283 